\title{
SOBEL EDGE DETECTION METHOD TO IDENTIFY AND QUANTIFY THE RISK FACTORS FOR DIABETIC FOOT ULCERS
}

\author{
Kunjam Nageswara Rao ${ }^{1}, \mathrm{P}_{\text {srinivasa }} \mathrm{Rao}^{2}$, Allam Appa Rao $^{3}$. Dr. G R Sridhar ${ }^{4}$ \\ ${ }^{1}$ Dept. of CS\&SE, Andhra university, Visakhapatnam, India, \\ kunjamnag@gmail.com \\ ${ }^{2}$ Dept. of CS\&SE, Andhrauniversity, Visakhapatnam, India, ${ }^{3}$ Director, Dr. CR Rao \\ AIMSCS, University of Hydeabad, Hyderabad, India, \\ 3apparaoallam@gmail.com, \\ ${ }^{4}$ Endocrine \& Diabetes centre, Visakhapatnam, India, \\ 5 sridharvizagegmail.com
}

\begin{abstract}
The purpose of this study is to identify and quantify the neurovascular risk factors for foot problems in patients with diabetes mellitus, which is a major public health concern these days. The most feared factor among the diabetic patients is lower extremity amputation. The sequence of events leading to amputation is initiated by ulceration combined with sensation loss. To prevent complications and amputations it is necessary to detect the foot at risk of plantar ulceration at an early stage of sensation loss. Doctors use imaging test to determine whether the plantar ulceration has spread to upper areas in the leg, and to evaluate the size and location of the ulcer. To locate the ulceration area and assess the severity, doctor's uses an imaging technology, such as ultrasound, fluoroscopy, a computed tomography (CT or CAT) scan, $x$-ray, or a magnetic resonance imaging (MRI) test, this procedure is called as an image-guided biopsy. To access the severity of foot ulcer, a new method is introduced, which is a popular edge detection method. This method is known as Sobel method, which uses edge detection function for derivative approximation to find edges. The Sobel edge method returns edges at those points where the gradient of the considered image is maximum, so the recognition of risk factors will be analyzed in efficient manner. Based on the severity of foot ulcer, preventive foot maintenance and regular foot examinations will take place in diabetes patients.
\end{abstract}

\section{KEYWORDS}

Diabetes, Foot Ulcer, Amputation, Plantar, Imaging.

\section{INTRODUCTION}

A problem for non-diabetics can be a significant risk factor for amputation in a diabetic patient such as some heart attacks, foot problems are common for people suffering with diabetes [1]. Foot ulcers, Infections and circulatory problems also take place for the people who are at risk for amputation. It is extremely hard to treat the blood vessel and nerve damage linked with diabetes, sometimes it can also lead to serious infections [2]. First the problem occurs in your feet and then it can spread up into the leg and to the whole body. Even a small injury, such as a cut can develop into an ulcer and causes a serious infection [3]. 
Amputation of a lower limb or lower-limb amputation (LLA), is a worst consequence of diabetes. People with diabetes are more than 10-15 times likely to have a LLA than non-diabetic individuals. The causation of LLA involves diseases like peripheral vascular disease (PVD), impaired wound healing, peripheral neuropathy, minor trauma, limited joint mobility. These factors lead to foot ulceration and finally cause amputation [4].

The most diabetic foot complications resulting in amputation start with skin ulcers. 85 percent of amputations can be prevented by early detection and appropriate treatment for these ulcers.

Careful inspection of the diabetic foot on a regular basis is one of the easiest and most effective measures [5]. Proper care of the diabetic foot requires realization of the most common risk factors for limb loss. These risk factors can be identified based on the specific aspects of the history and a brief but systematic examination of the foot.

Adequate debridement technique is the evaluation of a foot ulcer. The healthy bleeding edge cannot be revealed until and unless the debridement removes all the necrotic tissues and the surrounding callus. Patients and physicians may be surprised by the appearance of the newly debrided ulcer and often underestimate the need for debridement [5]. The topical debriding enzymes are very expensive and they are not conclusively shown to be beneficial.

After the debridement technique, to determine the involvement of underlying structures, such as joint capsule, tendon or bone the ulcer should be probed with sterile blunt instruments. Probing to bone is low sensitivity task but it is very simple and has a specific test for osteomyelitis [6]. To evaluate the ulcer for bone involvement and to look for soft tissue gas and foreign bodies Plainfilm radiographs should be obtained.

It is very difficult to differentiate the local soft tissue infection and inflammation from osteomyelitis. Radiolabelled leukocyte scans and three-phase bone scans are very expensive but these scans can help to establish an accurate diagnosis when these types of problem occur [7]. The presence or absence of ischemia and the involvement of underlying structures must be determined before an appropriate wound classification is made and a subsequent treatment plan should be implemented[8].

Prevention by early detection is the only solution. For this when it is in initial stage by usage of sobel edge detection method we can assess the vulnerability and start the treatment accordingly. This will save the patient from the far end procedure of amputation.

\section{IMAGE SEGMENTATION}

Segmentation sub divides an image into its constituents region or objects. The level to which the sub division is carry depends on the problem being solved. When the objects of interest in an application have been isolated then the segmentation should be stopped. For example, Interest lies in analyzing images of the products with the object of determining the presence or absence of specific anomalies, such as broken connection parts or missing components in automatic inspection of the assemblies.One of the most difficult task in the image processing is segmentation of non-trivial images. The segmentation accuracy determines the eventual success or failure of computerized analysis procedures. For this reason, to improve the probability of rugged segmentation, lot of care should be taken. In some situation, such as industrial inspection application, at least some measure of control over the environment is possible at times. In others, as in remote sensing, user control over image acquisition is limited principally to the choice imagining sensors Segmentations algorithms for monochrome images generally are based on one of two basic properties of image intensity values: discontinuity and similarity[9]. In first category the approach is to partition an image based on abrupt changes in intensity, such as edges 
in an image. For partitioning an image in to regions that are similar according to a set of predefined criteria the principle approaches in the second category.For detecting intensity discontinuities such as points, liens and edges, a number of methods are developed. Edge detection is one of the method which has been a staple of segmentation algorithms for many years. In addition to the edge detection detecting linear edge segments using methods based on the Hough transforms. The holding method is a fundamental approach in the segmentation that enjoys a significant degree of popularity, particularly in applications where speed is an important factor. A morphological approach to segmentation is called water shed segmentation. This approach is particularly attractive because it produces closed, well-define regions, behaves in a global fashion and provides a framework in which a priori knowledge about the images in particular application can be utilized to improve the segmentation results.

\section{EDGE DETECTION}

Edge detection is a process of restricting the pixel intensity transitions. The edge detection has been used by object recognition, target tracking, segmentation, and etc. Therefore, the edge detection is also one of the most important parts of image processing.

There mainly exist several edge detection methods which have been proposed for detecting transitions in images. Some of these methods are Sobel, Prewitt, Roberts and Canny. To detect sharp intensity variations, the early methods determine the best gradient operator. Applying derivative operator on images is the most commonly used method for detecting edges.

Derivative based approaches can be categorized into two groups, First order derivative method and Second order derivative method.

The First order derivative techniques depend on computing the gradient to several directions and combining the result of each gradient. The gradient magnitude value and the orientation are estimated using two differentiation masks. Here, an edge detection method (Sobel) is considered. This method is preferred compared to others methods in this work because of the simplicity and common uses. The Sobel edge detector method consists of two different masks, one vertical and one horizontal. These masks are generally used $3 \times 3$ matrices. In particular, the matrices which have $3 \times 3$ dimensions are used in mat lab (see, edge.m). The masks of the Sobel edge detection method are extended to $5 \times 5$ dimensions and they are constructed in this work. Finally a mat lab function which is called as Sobel $5 \times 5$ is developed using these new matrices[10]. 

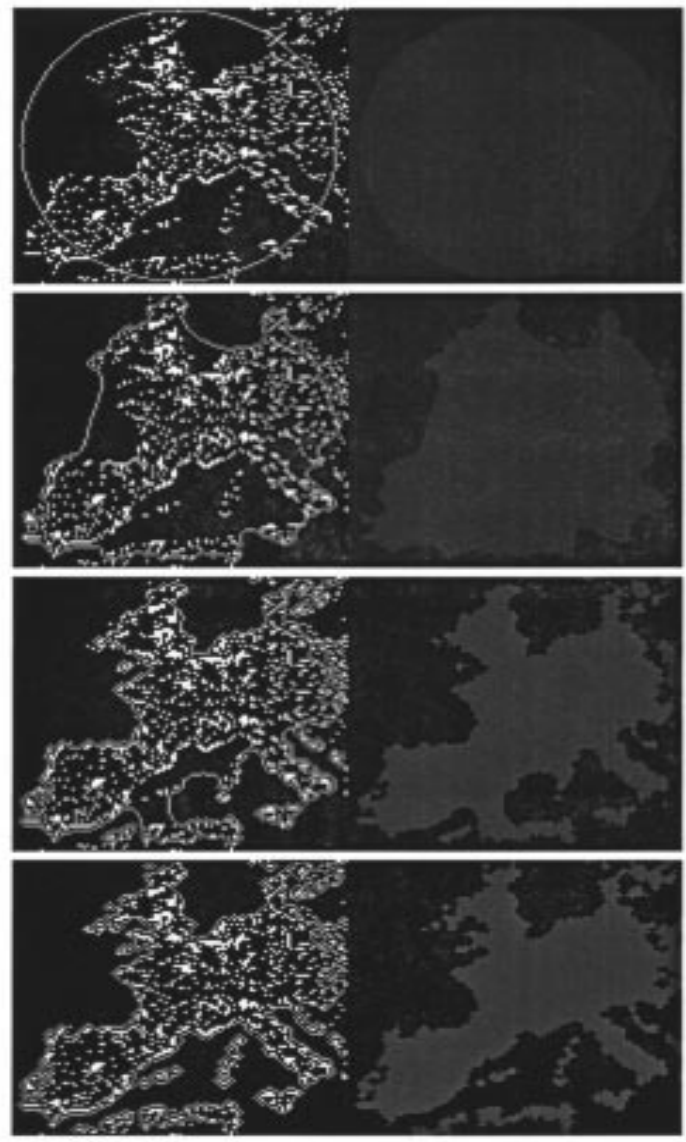

Fig. 1.

\section{EDGE DETECTION FUNCTION}

When Sobel masks is applied to an image in all directions then two new images are created, one image shows the horizontal response and the other shows the vertical response. These two images combine into a single image to determine the existence and location of edges in a picture. The combination of these two images explains that the square of created masks pixel estimate coincidence each other as coordinates are summed. Thus the new image on which edge pixels are located obtain the value which is the squared of the above summation. The threshold value in this above process is used to detect edge pixels. To find the edges using new matrices an algorithm and a mat lab function is developed which is called as Sobel $5 \times 5$ and is implemented in mat lab. This mat lab function requires a gray scale intensity image and a two-dimensional array.

\section{SOBEL EDGE DETECTION}

Standard Sobel operators, for a $3 \times 3$ neighborhoods, each simple central gradient estimate is vector sum of a pair of orthogonal vectors[1]. Each orthogonal vector is a directional derivative estimate multiplied by a unit vector which is specified by the direction of the derivative. The amounts to a vector sum of the 8 directional derivative vectors estimates vector sum of this simple gradient. Thus for a point on Cartesian grid and its eight neighbors having density values as shown: 
International Journal of Computer Science \& Information Technology (IJCSIT) Vol 5, No 1, February 2013

\begin{tabular}{|c|c|c|}
\hline$a$ & $b$ & $c$ \\
\hline$d$ & $e$ & $f$ \\
\hline$g$ & $h$ & $i$ \\
\hline
\end{tabular}

Fig. 2.

In the above matrix, the directional derivative estimate vector $G$ was defined such as density difference or distance to neighbor. This vector is determined such that the direction of derivative estimate vector $G$ will be given by the unit vector to the approximate neighbor. Note that, the neighbors group into antipodal pairs: $(a, i),(b, h),(c, g),(f, d)$. The vector sum for this gradient estimate:

$G=\frac{(i-g)}{R} \cdot \frac{[1,1]}{R}+\frac{(a-i)}{R} \cdot \frac{[-1,1]}{R}+(b-h) \cdot[0,1]+(f-d) \cdot[1,0]$

where, $R=\sqrt{2}$. This vestor is cbtain=c as

$G=[(c-g-a+i) / 2+j-d,(c-g+a-i) / 2+b-h]$

Here, this vector is muliplied by 2 because of repacing the sivize by 2 . The resulant formula is given as follows 's₹e, for detail [1]!:

$G^{\prime}=2 . G=\left[(c-g-a-i)+2 .\left(f-d^{\prime}\right),(c-g+a-i)+2 .(b-h i)\right]$

The fcllowing weighting functions for $x$ and $y$ comocnents were obtained of using the above vector.

\begin{tabular}{|c|c|c|}
\hline 1 & 0 & 1 \\
\hline-2 & 0 & 2 \\
\hline-1 & 0 & 1 \\
\hline
\end{tabular}

\begin{tabular}{|cc|c|}
\hline 1 & 2 & 1 \\
\hline 0 & 0 & 0 \\
\hline-1 & -2 & -1 \\
\hline
\end{tabular}

Fig. 3.

Now, we explain that the dimensions of the matrices are extended by using. The definition of the gradient can be used for $5 \times 5$ neighborhood. In this case, twelve directional gradients must be determined instead of four gradients. The following figure $5 \times 5$ neighborhood.

\begin{tabular}{|c|c|c|c|c|}
\hline $\mathrm{a}$ & $\mathrm{b}$ & $\mathrm{c}$ & $\mathrm{d}$ & $\mathrm{e}$ \\
\hline $\mathrm{f}$ & $\mathrm{g}$ & $\mathrm{h}$ & $\mathrm{i}$ & $\mathrm{j}$ \\
\hline $\mathrm{k}$ & $\mathrm{l}$ & $\mathrm{m}$ & $\mathrm{n}$ & $\mathrm{o}$ \\
\hline $\mathrm{p}$ & $\mathrm{r}$ & $\mathrm{s}$ & $\mathrm{t}$ & $\mathrm{u}$ \\
\hline $\mathrm{v}$ & $\mathrm{w}$ & $\mathrm{x}$ & $\mathrm{y}$ & $\mathrm{z}$ \\
\hline
\end{tabular}

Fig. 4.

The resultant vector $G^{\prime}$ (similar to the determination of Sobel $3 \times 3$ method) for $5 \times 5$ is given as follows:

$G^{\prime}=[20(n-l)+10(i-r-g+t+o-k)+5(e-v-a+z)+4(d-w-b+y)+8(\mathrm{j}-p-f+u)$,

$20(h-s)+10(i-r+g-t)+5 \times(e-v+a-z)$

$+4(\mathrm{j}-p+f-u)+8(d-w+b-y)]$ 
International Journal of Computer Science \& Information Technology (IJCSIT) Vol 5, No 1, February 2013

The horizontal and vertical masks are obtained by using the coefficients in this equation such as

\begin{tabular}{|c|c|c|c|c|}
\hline-5 & -4 & 0 & 4 & 5 \\
\hline-8 & -10 & 0 & 10 & 8 \\
\hline-10 & -20 & 0 & 20 & 10 \\
\hline-8 & -10 & 0 & 10 & 8 \\
\hline-5 & -4 & 0 & 4 & 5 \\
\hline
\end{tabular}

\begin{tabular}{|cc|c|c|c}
\hline 5 & 8 & 10 & 8 & 5 \\
\hline 4 & 10 & 20 & 10 & 4 \\
\hline 0 & 0 & 0 & 0 & 0 \\
\hline-4 & -10 & -20 & -10 & -4 \\
\hline-5 & -8 & -10 & -8 & -5 \\
\hline
\end{tabular}

\section{EXPERIMENTAL RESULTS}

We conclude this paper by saying the edge detection technology is the most popular Technology. This particular method is efficient than other edge detection methods like Laplacian of Gaussian operator and general morphological edge detection

algorithm. We successfully find the edges of the given medical image, and we can easily find the exact position of the ulcer as shown in fig.

Input Image :

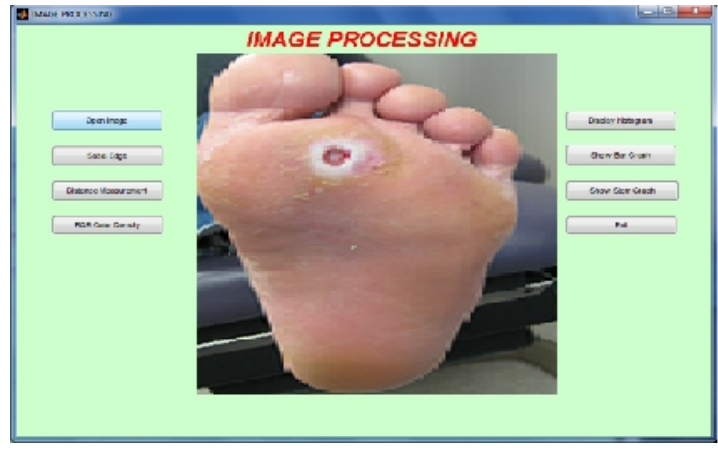

Fig. 5.

Output Edge

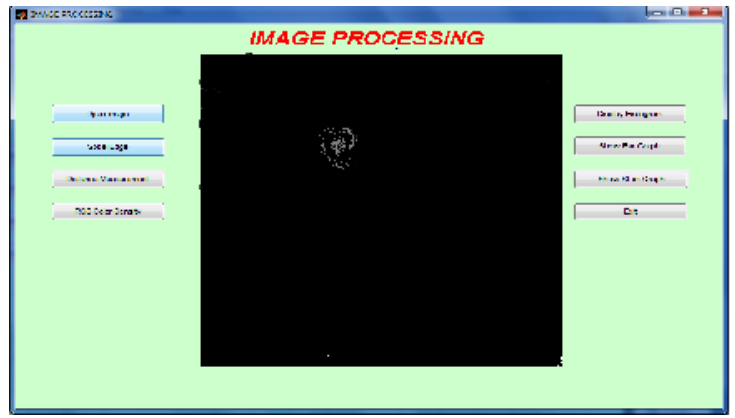

Fig. 6. 


\section{Distance Measurement}

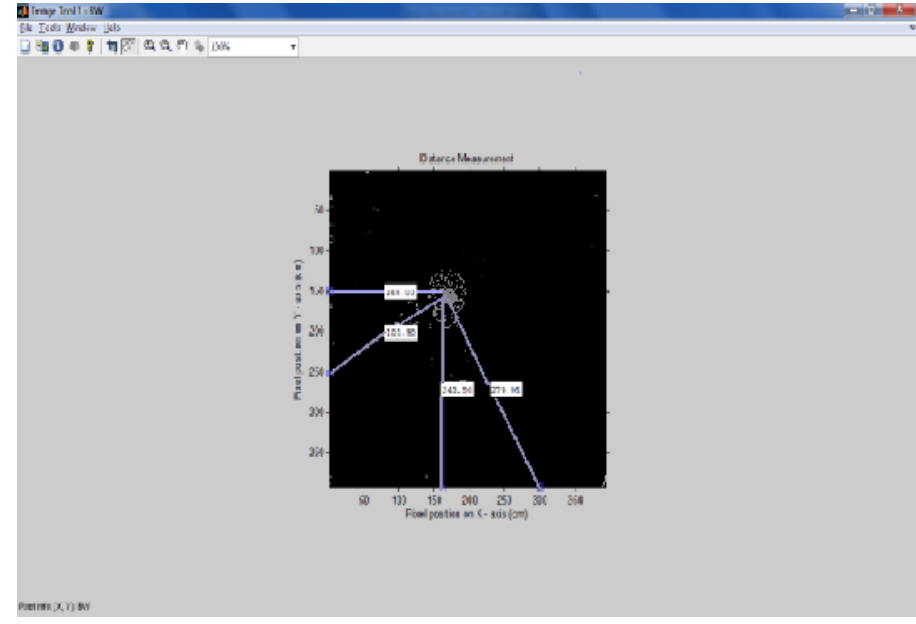

Fig. 7.

\section{CONCLUSION}

This system finds the edge of the image to observe the affected area of the ulceration. Which provides documentation of ulcer characteristics, including location, shape, and size of the wound This helps to the doctor while diagnose the patient for determination of the condition of the wound edges, wound bed, wound base, periwound skin, and exudates. This system takes a medical image for the detection of the ulceration in the lower extremity.

The system can be upgraded to detect the continuities in electronic circuits in PCB designing, Measuring tolerance measurements, Alignment - determining the orientation and position of a part, Identification - identifying a bar code, seven-segment display, meter, or written words.

Recognition of risk factors in preventive the foot maintenance and regular foot examinations which are essential in preventing foot ulcers in patients suffering with diabetes. When foot ulcers develop, a systematically applied regimen of diagnosis and classification coupled with early and appropriate treatment helps to reduce the tremendous personal and societal burden of diabetesrelated amputations.

\section{Acknowledgement}

The authors would like to thank Rafeal C. Gonzalez, Richard E. Woods, Steven L. Eddins, referees for their general and detailed comments and suggestions, which helped very much to improve the presentation of the paper. 
International Journal of Computer Science \& Information Technology (IJCSIT) Vol 5, No 1, February 2013

\section{REFERENCES}

[1] "Digital Image Processing Using MATLAB", Rafeal C. Gonzalez, Richard E. Woods, Steven L. Eddins, "Pearson Education"

[2] "Graphics GUIs with MATLAB - 2e", Patric Marchard, "CRC Press Inc."

[3] "Digital Image Processing", WILLIAM K. PRATT, "WILEY-INTERSCIENCE a John Wiley \& Sons, Inc., Publication"

[4] V. Caselles, R. Kimmel, and G. Sapiro, “On geodesic active contours,” Int. J. Comput. Vis., vol. 22, no. 1, pp. 61-79, 1997.

[5] Caputo GM, Cavanagh PR, Ulbrecht JS, Gibbons GW, Karchmer AW. Assessment and management of foot disease in patients with diabetes. N Engl J Med. 1994.

[6] Lavery LA, Armstrong DG, Harkless LB. Classification of diabetic foot wounds. J Foot Ankle Surg. 1996.

[7] Armstrong DG, Lavery LA, Harkless LB. Treatment-based classification system for assessment and care of diabetic feet. J Am Podiatr Med Assoc. 1996; 86:311-6.

[8] Adler AI, Ahroni JH, Boyko EJ, Smith DG: Lower-extremity amputation in diabetes: the independent effects of peripheral vascular disease, sensory neuropathy, and foot ulcers. Diabetes Care22: 10291035, 1999.

[9] Val GD, Kriegsman DM, Assendelft WJ. Patient education for preventing diabetic foot ulceration. Cochrane Database Syst Rev 2001;

[10] Boulton AJ, Vileikyte L, Ragnarson-Tennvall G, Apelqvist J. The global burden of diabetic foot disease. Lancet. 2005; 